Manuelle Medizin 2008 · 46:204-204

DOI 10.1007/s00337-008-0621-6

Online publiziert: 23. Juli 2008

(c) Springer Medizin Verlag 2008

\author{
R. Kayser ${ }^{1} \cdot$ L. Beyer ${ }^{2}$ \\ ${ }^{1}$ Klink und Poliklinik für Orthopädie und Orthopädische Chirurgie, \\ Universitätsklinikum Greifswald \\ 2 Ärztehaus Mitte, Jena
}

\title{
Wissenschaftlichkeit in der manuellen Medizin oder die Quadratur des Kreises?
}

dener wissenschaftlicher Erkenntnisse und andererseits die Veröffentlichung eines Literaturverzeichnisses, welches dem praktisch tätigen Manualmediziner als argumentative Hilfe zur Verfügung stehen soll. Ziel eines Reviews ist es nicht, persönliche Erfahrungen einzubringen oder persönliche Meinungen zu diskutieren. Nach Meinung der Herausgeber ist das genannte Verfahren und die Publikation von wissenschaftlichen Studien, die einem Peer-Review-Verfahren standhalten, der einzige Weg, die manuelle Medizin als Teil der medizinischen Wissenschaften darzustellen und damit auf lange Sicht überlebensfähig zu machen.

\section{$>$ Auch künftig wird es Raum für Kasuistiken, Erfahrungsberichte und Meinungen geben}

Zweifelsohne ist es nicht unser Ziel, ausschließlich wissenschaftliche Erkenntnisse im Sinne der evidenzbasierten Medizin zu veröffentlichen. Vielmehr steht in der Zeitschrift in einem separaten Teil genügend Raum zur Verfügung, Kasuistiken, Erfahrungsberichte, aber auch Meinungen und Statements abzudrucken, soweit sie nach Meinung der Herausgeber von allgemeinem Interesse sind. Wie dicht hierbei die Grenzen sind und wie unterschiedlich die von uns beauftragten Reviewer auch die Qualität der Artikel einschätzen, ist bei der Publikation des einen oder anderen Artikels sicher zu bemerken. Um die Listung der Zeitschrift erreichen zu können und die Vermischung der unterschiedlichen Rubriken künftig möglichst zu vermeiden, haben wir uns entschlossen, dem Peer-Review-Verfahren ein Herausgebervotum anzuschlie- ßen, welches vor der Publikation eines eingereichten Artikels im wissenschaftlichen Teil der Zeitschrift notwendig ist. Wir sind ganz sicher, dass nur die wissenschaftliche Beurteilung unserer täglichen Arbeit eine Weiterentwicklung des Fachgebietes manuelle Medizin gewährleisten kann. Dies kann gern auch in Form von Versorgungsforschung und in der Evaluierung unserer Behandlungsergebnisse erfolgen. Dieses ist, wie oft gezeigt, auch unter Praxisbedingungen gut möglich. Hierzu müssen jedoch die Methoden der wissenschaftlichen Arbeit eingehalten werden.

Wir dürfen Sie abschießend nochmals sehr herzlich einladen, Ihre wissenschaftlichen Kenntnisse und Erfahrungen in Bezug auf die manuelle Medizin in unserer Zeitschrift zu veröffentlichen. Vonseiten der Herausgeber und der DGMM werden Sie jede nur mögliche Unterstützung erhalten. Wir freuen uns auf die Einsendung Ihrer Manuskripte und auf eine weiter fruchtbringende Diskussion im Interesse der gemeinsamen Sache.

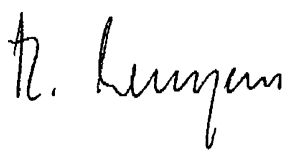

PD Dr. med. R. Kayser

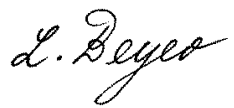

Prof. Dr. med. L. Beyer

\section{Korrespondenzadresse \\ PD Dr. R. Kayser}

Klink und Poliklinik für Orthopädie und

Orthopädische Chirurgie,

Universitätsklinikum Greifswald

Ferdinand-Sauerbruch-Str.,

17475 Greifswald

ralph.kayser@uni-greifswald.de 